\title{
TeV Scale Implications of Non Commutative Space time in Laboratory Frame with Polarized Beams
}

\author{
Sumit K. Garg, $1, *$ T. Shreecharan, ${ }^{2,+}$ P. K. Das, ${ }^{3,+}$ \\ N. G. Deshpande,, \& and G. Rajasekaran $5,6, q$ \\ ${ }^{1}$ Centre for High Energy Physics, Indian Institute of Science, Bangalore 560 012, India \\ ${ }^{2}$ School of Physics, University of Hyderabad, Hyderabad 500 046, India \\ ${ }^{3}$ Department of Physics, Birla Institute of Technology and Science-Pilani, \\ K.K. Birla Goa campus, NH-17B, Zuarinagar, Goa 403 726, India \\ ${ }^{4}$ Institute of Theoretical Science, University of Oregon, Eugene, Oregon 97403, USA \\ ${ }^{5}$ The Institute of Mathematical Sciences, \\ C.I.T. Campus, Taramani, Chennai 600 113, India \\ 6 Chennai Mathematical Institute, Siruseri 603103, India
}

\begin{abstract}
We analyze $e^{+} e^{-} \rightarrow \gamma \gamma, e^{-} \gamma \rightarrow e^{-} \gamma$ and $\gamma \gamma \rightarrow e^{+} e^{-}$processes within the Seiberg-Witten expanded noncommutative scenario using polarized beams. With unpolarized beams the leading order effects of non commutativity starts from second order in non commutative(NC) parameter i.e. $O\left(\Theta^{2}\right)$, while with polarized beams these corrections appear at first order $(O(\Theta))$ in cross section. The corrections in Compton case can probe the magnetic component $\left(\vec{\Theta}_{B}\right)$ while in Pair production and Pair annihilation probe the electric component $\left(\vec{\Theta}_{E}\right)$ of NC parameter. We include the effects of earth rotation in our analysis. This study is done by investigating the effects of non commutativity on different time averaged cross section observables. The results which also depends on the position of the collider, can provide clear and distinct signatures of the model testable at the International Linear Collider(ILC).
\end{abstract}

*Electronic address: sumit@cts.iisc.ernet.in

${ }^{\dagger}$ Electronic address: shreet@imsc.res.in

‡Electronic address: pdas@bits-goa.ac.in

$\S$ Electronic address: desh@uoregon.edu

"Electronic address: graj@imsc.res.in 


\section{INTRODUCTION}

Field theories defined on a non commutative(NC) space time have been extensively studied in the past few years. This idea received much attention due to its possible connection with quantum gravity and because of its natural origin in string theories. Infact Seiberg and Witten[1] described how NC gauge theory can emerge as a low energy manifestation of string theory.

However the original idea was considered long time ago when non commutativity of Minkowski Space-time was assumed as a natural extension of Heisenberg's positionmomentum non commutativity in quantum mechanics. In early work of Snyder[2] the non commutativity of space time was suggested as a possible cure for ultraviolet divergences in Quantum field theory(QFT). However this viewpoint was largely ignored mainly because at that time the renormalization techniques in QFT met great success predicting quite accurately the numerical values for physical observables in quantum electrodynamics(QED).

This field got renewed attention in 2000 after the work of Seiberg and Witten[1]. They showed that the dynamics of open strings ending on D-branes in a background field can be described by a non commutative quantum field theory(NCQFT). They also gave the explicit mapping between NC and ordinary gauge theories which is famously known as the SeibergWitten Map(SWM). This mapping in turn emerged as the roadmap for investigation of the gauge theories like Standard Model in non commutative space time.

Parallel to this development another approach, based on the Moyal-Weyl (MW) star product Eq.(2), also became popular. It was very soon realized that NC field theories constructed via this approach are plagued by the so called UV/IR effect [3, 4] wherein additional divergences appeared in the infrared that were not present in the commutative theory.

Apart from these developments NC theories are also supposed to shed some light on the quantization of space time (i.e. quantum theory of gravity) in the context of string theory. Thus it served as one of the major motivation for the intense activity in this area among string theorists. These ideas may lead to the possibility of making the Standard Model(SM) consistent with quantum gravity. In this context it worth pointing out that that the UV/IR effect plays an important role in determining what the UV theory might be. In other words a high energy theory might show up consequences at low energy which are will 
within experimental reach. This has been discussed, within the context of MW NC setting, in the works [5- 8 ].

Hence keeping in mind the above motivations it is reasonable to examine field theories, and in particular the standard model of particle physics on non commutative space time. We adopt an approach based on SWM popularized by the Munich group[9 16].

The reason why NC collider phenomenology is interesting, comes from the fact that the scale of non commutativity could be as low as a few $\mathrm{TeV}$, which can be explored at the present or the future colliders. This led to a great deal of interest in phenomenology of the NCSM with SWM. Many phenomenological signatures have been studied by different research groups. These works were mainly done [17-34] with unpolarized beams with leading corrections to SM starting from $O\left(\Theta^{2}\right)$. However few studies [14, 35, 36] are also done with corrections at the $O(\Theta)$ in cross section. Previous studies for processes considered here are often incomplete because $O\left(\Theta^{2}\right)$ contribution to scattering amplitudes requires Feynman rules to $O\left(\Theta^{2}\right)$ which were not included, and these terms are known to have intrinsic ambiguities and thus making the calculations indefinite.

In this work we have calculated $(O(\Theta))$ corrections for Compton, pair annihilation and pair production while keeping only one initial beam polarization $\left(e^{-}\right.$in Compton and pair annihilation and $\gamma$ in pair production). We have also taken into account the effect of earth's rotation [37-40] on observable signals of NC. The effects of Non commutativity is studied on various time averaged observables to determine the magnitude and direction of NC parameter.

We have looked at the possible implications of the NC corrections for phenomenology at the International Linear Collider(ILC) [41, 42]. In addition to $e^{-} e^{+}$programme, linear colliders also provide a unique opportunity to study $\gamma \gamma$ and $\gamma e$ interactions at energies and luminosities comparable to initial electron-positron beam. Intense beam of high energy photons can be obtained using Compton backscattering of laser light off the high energy electrons. If these beams become available at future Linear Colliders then it will serve as crucial test of NCQED in the processes we discuss.

The rest of the paper is organized as follows. In section II, we will briefly describe the mathematical description of non commutative space time. In section III, we give the cross section details for the mentioned processes. In section IV, we will present our numerical results. Finally we conclude with a section on our results and a discussion. 


\section{NON COMMUTATIVE STANDARD MODEL}

The idea of non commutative space is the generalization of quantum mechanics in which the canonical position and momentum variables $x_{i}, p_{j}$ are replaced with hermitian operators $\hat{x}_{i}, \hat{p}_{j}$ which obey the famous Heisenberg commutation relation

$$
\left[\hat{x}_{i}, \hat{p}_{j}\right]=\frac{i h}{(2 \pi)} \delta_{i j}
$$

So just like the qunatization of Classical Phase space, a non commutative space time coordinates $x^{\mu}$ are replaced by the Hermitian generators $\hat{x}^{\mu}$ which obeys the NC commutation relations $(\mu, \nu=0,1,2,3)$

$$
\left[\hat{x}^{\mu}, \hat{x}^{\nu}\right]=i \Theta^{\mu \nu}
$$

where $\Theta^{\mu \nu}$ is antisymmetric constant matrix with units of (Length) ${ }^{2}$. Thus in general one can consider two cases: first with space-space non commutativity associated with $\Theta^{i j}, \mathrm{i}, \mathrm{j}=1,2,3$ (known as magnetic components) and second with space-time non commutative related with $\Theta^{0 i}$ (electric components).

In field theory context, we realize Eq.(1) by using Moyal-Weyl(MW) «-product, defined by

$$
(f \star g)(x)=\left.\exp \left(\frac{i}{2} \Theta_{\mu \nu} \partial_{x}^{\mu} \partial_{y}^{\nu}\right) f(x) g(y)\right|_{y=x} .
$$

Thus one can construct NC field theories by replacing the ordinary products of fields with the corresponding star products. This replacement affects only the interaction parts and not the free field theory. In case of gauge theories, this approach is only consistent for $U(N)$ gauge theories and only a single eigenvalue is allowed for the charge operator.

To construct the NC extension of the standard model (SM) [11, 12, 15, 16], which uses the same gauge group and particle content, or for that matter any other gauge theory, be it abelian or non-abelian, one expands the NC gauge fields in non linear power series of $\Theta$ $\lfloor 1,9,10]$ :

$$
\begin{aligned}
& \lambda_{\alpha}(x, \Theta)=\alpha(x)+\Theta^{\mu \nu} \lambda_{\mu \nu}^{(1)}(x ; \alpha)+\Theta^{\mu \nu} \Theta^{\eta \sigma} \lambda_{\mu \nu \eta \sigma}^{(2)}(x ; \alpha)+\cdots \\
& A_{\rho}(x, \Theta)=A_{\rho}(x)+\Theta^{\mu \nu} A_{\mu \nu \rho}^{(1)}(x)+\Theta^{\mu \nu} \Theta^{\eta \sigma} A_{\mu \nu \eta \sigma \rho}^{(2)}(x)+\cdots
\end{aligned}
$$

At face value it can be seen from the above map that SW approach leads to a field theory with an infinite number of vertices and Feynman graphs thereby leading to an uncontrolled 


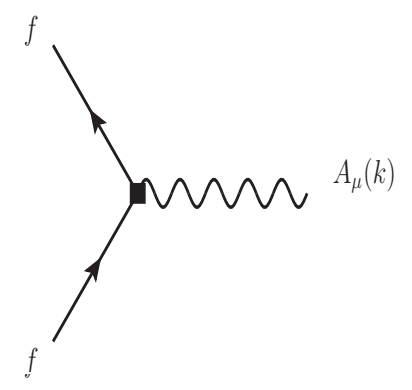

FIG. 1: 3-point NC $e^{-} e^{-} \gamma$ vertex

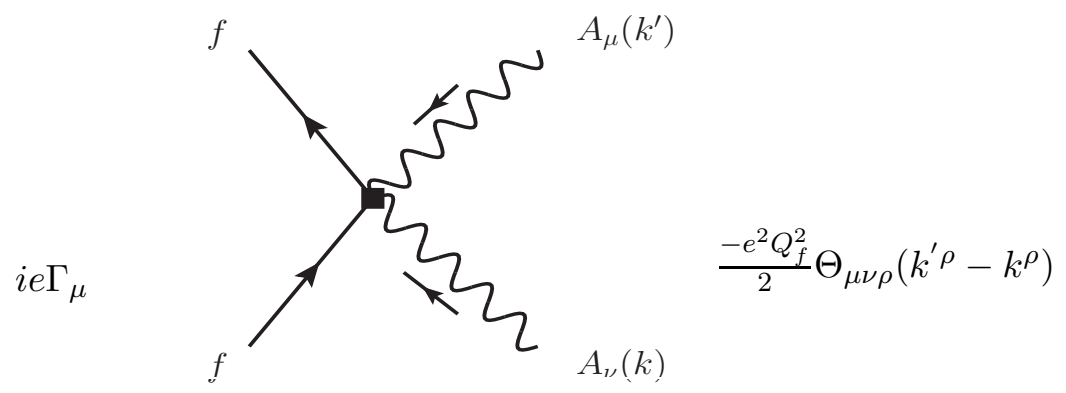

FIG. 2: 4-point $\mathrm{NC} e^{-} e^{-} \gamma \gamma$ vertex, $Q_{f}$ denotes the electric charge of fermion $\mathrm{f}$.

degree of divergence inturn giving an impression of complete failure of perturbative renormalization. But over the years a number of studies have shown that it is possible to construct anomaly free, renormalizable, and effective theories at one loop and first order in $\Theta[43-$ 51]. Before we provide the Feynman rules it must be mentioned that the celebrated IR/UV mixing, discussed in the earlier section, does not exist in the above $\Theta$ expanded approach. Though this is not a drawback in the scales of our interest there do exist certain phenomena that require all orders of the NC parameter be retained. This led to the so called $\Theta$-exact approach, that is from the exact solutions of the SW equations. The phenomenological consequences of this have been explored in [52, 53].

The above mentioned studies provide confidence in using the using NC SW expanded SM for phenomenological purposes. The Feynman rules for the NCSM have been worked out in [11, 15, 16] and the ones relevant for this work specifically minimal NCSM are given below. The rules for the 3-point $e^{-} e^{-} \gamma$ vertex (Fig.1) and the 4-point $e^{-} e^{-} \gamma \gamma$ vertex (Fig.2) are [15]

$$
\Gamma^{\mu}=\gamma^{\mu}-\frac{i}{2}\left\{\left(p_{\text {out }} \Theta p_{\text {in }}\right) \gamma^{\mu}-\left(p_{\text {out }} \Theta\right)^{\mu}\left(\not p_{\text {in }}-m\right)-\left(\not p_{\text {out }}-m\right)\left(\Theta p_{\text {in }}\right)^{\mu}\right\}
$$

and

$$
\begin{aligned}
\Theta^{\mu \nu \rho}\left(k^{\prime}-k\right)_{\rho} & =\Theta^{\mu \nu} \gamma^{\rho}\left(k^{\prime}-k\right)_{\rho}+\Theta^{\nu \rho} \gamma^{\mu}\left(k^{\prime}-k\right)_{\rho}+\Theta^{\rho \mu} \gamma^{\nu}\left(k^{\prime}-k\right)_{\rho} \\
& =\Theta^{\mu \nu}\left(\not k^{\prime}-\not k\right)+\gamma^{\mu}\left(\Theta\left(k^{\prime}-k\right)\right)^{\nu}-\left(\Theta\left(k^{\prime}-k\right)\right)^{\mu} \gamma^{\nu}
\end{aligned}
$$

respectively. Here $p_{i n}$ is the incoming and $p_{\text {out }}$ is outgoing momentum of fermion at vertex with $\left(p_{\text {out }} \Theta p_{\text {in }}\right) \equiv p_{\mu} \Theta^{\mu \nu} p_{\nu},(p \Theta)^{\nu} \equiv p_{\mu} \Theta^{\mu \nu}$ and $(\Theta p)^{\mu} \equiv \Theta^{\mu \nu} p_{\nu}$. However here we will work in the massless limit of electron and positron. 


\section{CROSS SECTIONS IN THE LABORATORY FRAME}

In this section we will give the calculational details of our work, first starting from the Compton scattering case.

This process in NCQED proceed at the tree level by the following diagrams(Fig.3). The first two diagrams also appears in pure QED while 3rd one arises just because of non commutative nature of space time and is a contact interaction.

The Feynman amplitudes for these diagrams with initial $e^{-}$beam polarization in NCQED are given by expressions:

$$
\begin{aligned}
i \mathcal{M}_{a}= & {\left[\bar{u}\left(p^{\prime}\right) H_{L, R}\left(i e \Gamma^{\mu}\right) \frac{i}{\not p+\not k}\left(i e \Gamma^{\nu}\right) u(p)\right] \epsilon_{r^{\prime} \mu}^{*}\left(k^{\prime}\right) \epsilon_{r_{\nu}}(k) } \\
= & \mathcal{M}_{1 a} \epsilon_{r^{\prime}}^{*}\left(k^{\prime}\right) \epsilon_{r}(k) \\
= & \left\{-e^{2} T_{11}^{\mu \nu}+\frac{i e^{2}}{2}\left(\left(T_{12}^{\Theta}\right)^{\mu \nu}+\left(T_{13}^{\Theta}\right)^{\mu \nu}\right)+O\left(\Theta^{2}\right)\right\} \epsilon_{r^{\prime} \mu}^{*}\left(k^{\prime}\right) \epsilon_{r_{\nu}}(k) \\
i \mathcal{M}_{b}= & {\left[\bar{u}\left(p^{\prime}\right) H_{L, R}\left(i e \Gamma^{\nu}\right) \frac{i}{\not p-\not k^{\prime}}\left(i e \Gamma^{\mu}\right) u(p)\right] \epsilon_{r^{\prime} \mu}^{*}\left(k^{\prime}\right) \epsilon_{r_{\nu}}(k) } \\
= & \mathcal{M}_{1 b} \epsilon_{r^{\prime}}^{*}\left(k^{\prime}\right) \epsilon_{r}(k) \\
= & \left\{-e^{2} T_{21}^{\nu \mu}+\frac{i e^{2}}{2}\left(\left(T_{22}^{\Theta}\right)^{\nu \mu}+\left(T_{23}^{\Theta}\right)^{\nu \mu}\right)+O\left(\Theta^{2}\right)\right\} \epsilon_{r^{\prime} \mu}^{*}\left(k^{\prime}\right) \epsilon_{r_{\nu}}(k) \\
& =\left\{\frac{i e^{2}}{2}\left(-i T^{\Theta}\right)^{\mu \nu}\right\} \epsilon_{r^{\prime} \mu}^{*}\left(k^{\prime}\right) \epsilon_{r_{\nu}}(k) \\
& =\mathcal{M}_{1 \epsilon^{\prime}} \epsilon_{r^{\prime}}\left(k^{\prime}\right) \epsilon_{r}(k) \\
= & {\left[\bar{u}\left(p^{\prime}\right)\left\{\frac{e^{2}}{2} \Theta^{\mu \nu \rho}\left(k^{\prime}+k\right)_{\rho}\right\} H_{(L, R)} u(p)\right] \epsilon_{r^{\prime} \mu}^{*}\left(k^{\prime}\right) \epsilon_{r_{\nu}}(k) } \\
& \\
& \\
& \\
& \\
&
\end{aligned}
$$

Here $u(p), \bar{u}\left(p^{\prime}\right)$ denote the spinor for incoming electron and outgoing electron respectively. $\epsilon_{r_{\nu}}(k), \epsilon_{r^{\prime} \mu}^{*}\left(k^{\prime}\right)$ are polarization vectors for incoming photon(polarization $\mathrm{r}$ ) and outgoing photon(polarization $\left.r^{\prime}\right)$ respectively. $H_{R, L}\left(\frac{1 \pm \gamma_{5}}{2}\right)$ are projection operators for right 

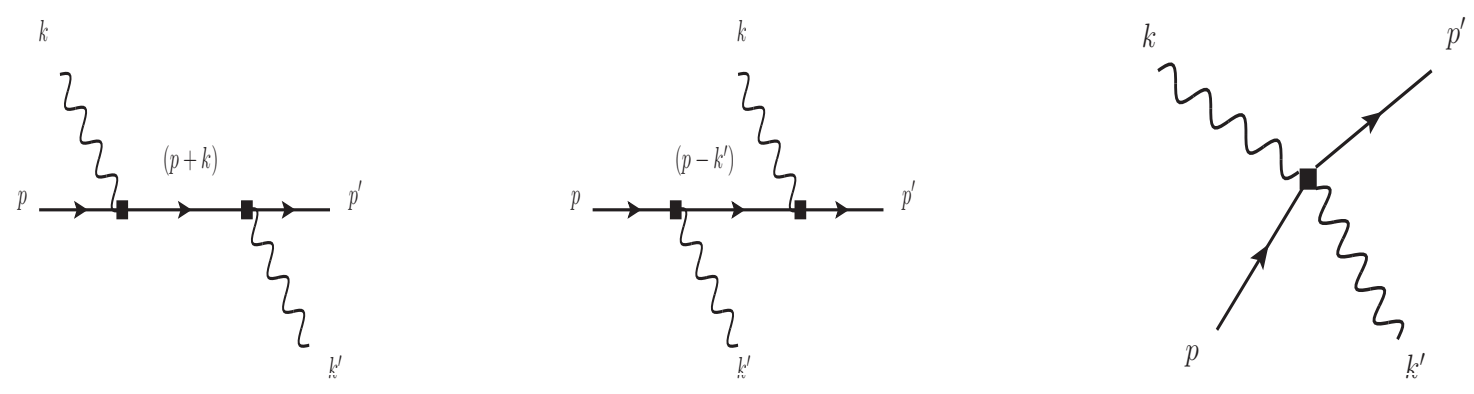

FIG. 3: Feynman diagrams for Compton scattering in NCQED.

and left helicity eigenstates of the electron.

Thus the total amplitude for the above process is given by the expression

$$
\begin{aligned}
\mathcal{M} & =i \mathcal{M}_{a}+i \mathcal{M}_{b}+i \mathcal{M}_{c} \\
& =\left(\mathcal{M}_{1 a}+\mathcal{M}_{1 b}+\mathcal{M}_{1 c}\right) \epsilon_{r^{\prime}}^{*}\left(k^{\prime}\right) \epsilon_{r}(k) \\
& =\left\{-e^{2}\left(T_{11}^{\mu \nu}+T_{21}^{\nu \mu}\right)+\frac{i e^{2}}{2}\left(\left(T_{12}^{\Theta}+T_{13}^{\Theta}\right)^{\mu \nu}+\left(T_{22}^{\Theta}+T_{23}^{\Theta}\right)^{\nu \mu}-i\left(T^{\Theta}\right)^{\mu \nu}\right)+O\left(\Theta^{2}\right)\right\} \epsilon_{r^{\prime} \mu}^{*}\left(k^{\prime}\right) \epsilon_{r_{\nu}}(k)
\end{aligned}
$$

The expressions of various T's are given in Appendix A.

It is clear from above expression that interference between SM and NC terms can provide $O(\Theta)$ corrections to cross section. However one will have to compensate for the imaginary factor i to get non vanishing $O(\Theta)$ correction in cross section. This can be done by taking initial beam polarized which will then generate a factor $i \epsilon^{\mu \nu \lambda \sigma}$ in Dirac traces and thus will produce non vanishing $\mathrm{NC}$ effects at leading order.

Since non commutative parameter is considered as fundamental constant in nature, so its direction is fixed in some non rotating coordinate system(can be taken to be celestial sphere). However the experiment is done in laboratory coordinate system which is rotating with earth's rotation. So one should take into account these rotation effects on $\Theta^{\mu \nu}$ in this frame before moving towards the phenomenological investigations.

These effects were considered in many previous studies[37-40] but we shall follow reference [39]. In the laboratory coordinate system, the orthonormal basis of the non ro- 

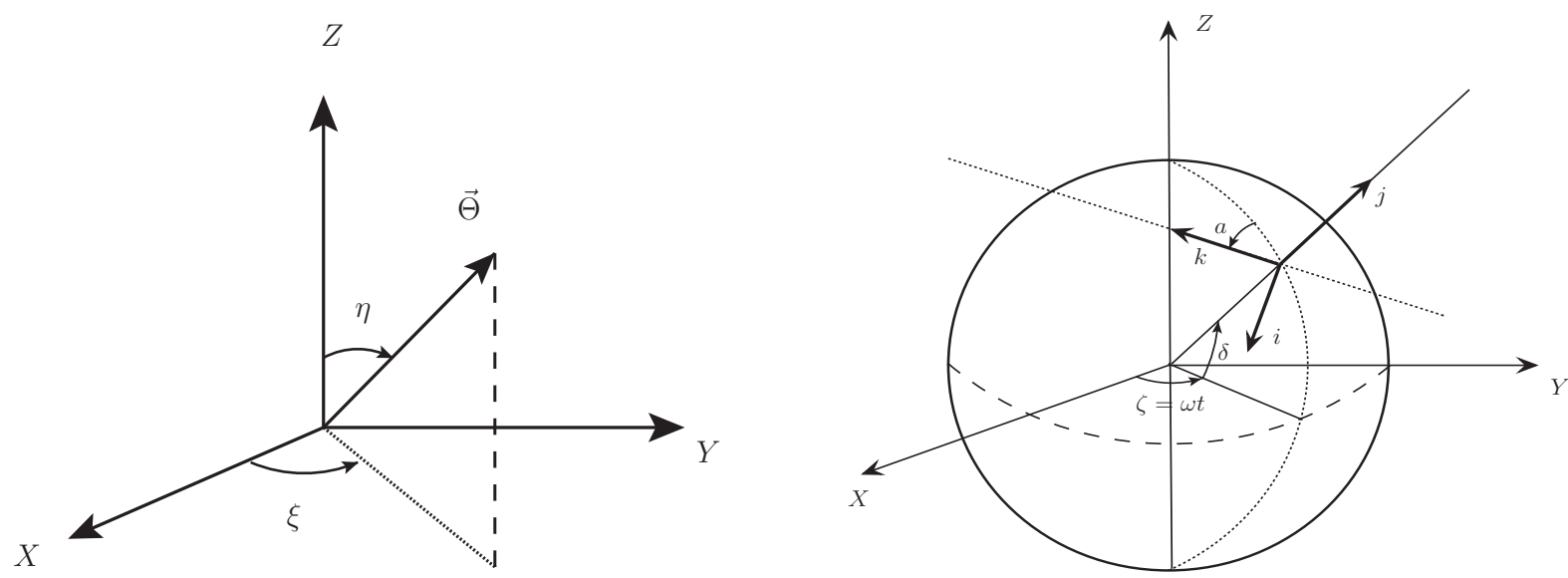

FIG. 4: X-Y-Z is the primary coordinate system while $\{\hat{i}-\hat{j}-\hat{k}\}$ are unit vectors pertaining to the laboratory coordinate system. The direction of $\vec{\Theta}$ is defined by angles $\eta$ and $\xi$.

tating(primary) coordinate $\operatorname{system}\left(\hat{i}_{X}-\hat{j}_{Y}-\hat{k}_{Z}\right)$ can be written as(see Fig.4)

$$
\hat{i}_{X}=\left(\begin{array}{c}
c_{a} s_{\zeta}+s_{\delta} s_{a} c_{\zeta} \\
c_{\delta} c_{\zeta} \\
s_{a} s_{\zeta}-s_{\delta} c_{a} c_{\zeta}
\end{array}\right), \hat{j}_{Y}=\left(\begin{array}{c}
-c_{a} c_{\zeta}+s_{\delta} s_{a} s_{\zeta} \\
c_{\delta} s_{\zeta} \\
-s_{a} c_{\zeta}-s_{\delta} c_{a} s_{\zeta}
\end{array}\right), \hat{k}_{Z}=\left(\begin{array}{c}
-c_{\delta} s_{a} \\
s_{\delta} \\
c_{\delta} c_{a}
\end{array}\right) .
$$

Here we have used the abberivations $c_{\alpha}=\cos \alpha, s_{\alpha}=\sin \alpha$ etc. $(\delta, a)$ defines the location of experiment with $-\pi / 2 \leq \delta \leq \pi / 2$ and $0 \leq a \leq 2 \pi$. Due to earth's rotation angle $\zeta$ increases with time and detector comes to its original position after a cycle of one day so one can define $\zeta=\omega t$ with $\omega=2 \pi / T_{\text {day }}$ where $T_{\text {day }}=23 h 56 m 4.09053 s$.

Thus the NC parameter in the Laboratory frame is given by electric and magnetic components

$$
\begin{aligned}
& \vec{\Theta}_{E}=\Theta_{E}\left(\sin \eta_{E} \cos \xi_{E} \hat{i}_{X}+\sin \eta_{E} \sin \xi_{E} \hat{j}_{Y}+\cos \eta_{E} \hat{k}_{Z}\right) \\
& \vec{\Theta}_{B}=\Theta_{B}\left(\sin \eta_{B} \cos \xi_{B} \hat{i}_{X}+\sin \eta_{B} \sin \xi_{B} \hat{j}_{Y}+\cos \eta_{B} \hat{k}_{Z}\right)
\end{aligned}
$$

with

$$
\vec{\Theta}_{E}=\left(\Theta^{01}, \Theta^{02}, \Theta^{03}\right) \quad \vec{\Theta}_{B}=\left(\Theta^{23}, \Theta^{31}, \Theta^{12}\right)
$$

and 


$$
\Theta_{E}=\left|\vec{\Theta}_{E}\right|=1 / \Lambda_{E}^{2} \quad \Theta_{B}=\left|\vec{\Theta}_{B}\right|=1 / \Lambda_{B}^{2}
$$

Here $(\eta, \xi)$ specifies the direction of NC parameter $\left(\Theta^{\mu \nu}\right)$ w.r.t primary coordinates system with $0 \leq \eta \leq \pi, 0 \leq \xi \leq 2 \pi$. $\Theta_{E}$ and $\Theta_{B}$ are absolute values of its electric and magnetic components with corresponding scales $\Lambda_{E}$ and $\Lambda_{B}$ respectively. One in general can probe them separately in different processes.

Using these definitions one can evaluate the various cross section observables with either standard Trace technique or by helicity amplitude method. We follow here the Trace technique and various traces in cross sections are evulated by using the Mathematica Package FeynCalc[54]. The results are cross checked in symbolic manipulation program FORM[55]. Few details of the calculation are given in appendix A.

Thus in the Center of Mass frame $\left(A(p)+B(k)-->A\left(p^{\prime}\right)+B\left(k^{\prime}\right)\right)$

$$
\begin{aligned}
p^{\mu} & =\frac{\sqrt{s}}{2}\{1,0,0,1\} \\
k^{\mu} & =\frac{\sqrt{s}}{2}\{1,0,0,-1\} \\
p^{\mu^{\prime}} & =\frac{\sqrt{s}}{2}\{1, \sin \theta \cos \phi, \sin \theta \sin \phi, \cos \theta\} \\
k^{\mu^{\prime}} & =\frac{\sqrt{s}}{2}\{1,-\sin \theta \cos \phi,-\sin \theta \sin \phi,-\cos \theta\}
\end{aligned}
$$

where $\theta$ is the polar angle and $\phi$ is the azimuthal angle, with initial beam direction chosen as the z-axis.

The time dependence in cross section enters through the NC parameter $\vec{\Theta}$ which changes with change of angle $\zeta$ because of earth's rotation. The final cross section formulae for different cases are given by:

\section{For Compton Scattering:}

The differential cross section with keeping only incoming electron beam in Right polarized state is given by

$$
\left(\frac{d \sigma}{d \Omega}\right)_{\overrightarrow{\Theta_{B}}}=\frac{\alpha^{2}}{8 s}\left[\left(2 \cos \theta+\cos ^{2} \theta+5\right) \sec ^{2} \frac{\theta}{2}+\bar{s}_{B}\left\{L_{1}^{\theta}\left(\Theta^{23} \cos \phi+\Theta^{31} \sin \phi\right)+L_{2}^{\theta} \Theta^{12}\right\}\right]
$$


where

$$
\bar{s}_{B}=\frac{s}{\Lambda_{B}^{2}}, \quad L_{1}^{\theta}=4 \sin ^{2} \frac{\theta}{2}\left(1+\cos ^{2} \frac{\theta}{2}\right) \tan \frac{\theta}{2} ; \quad L_{2}^{\theta}=4 \sin ^{2} \frac{\theta}{2}\left(1+\cos ^{2} \frac{\theta}{2}\right)
$$

Now the cross sections for Pair annihilation and Pair Creation are easy to evaluate since they are related to the Compton by crossing symmetry. The cross section for Pair annihilation can be calculated by substituting $p^{\prime} \rightarrow-p^{\prime}, k \rightarrow-k$ while for Pair production can be found by inserting $p \rightarrow-p, k^{\prime} \rightarrow-k^{\prime}$ in Compton trace expressions. The differential cross section formulae for these two cases are given as:

\section{For Pair Annihilation:}

The differential cross section with keeping only incoming electron beam in Right polarized state is given by

$$
\left(\frac{d \sigma}{d \Omega}\right)_{\overrightarrow{\Theta_{E}}}=\frac{\alpha^{2}}{s}\left[\left(1+\cos ^{2} \theta\right) \csc ^{2} \theta-\bar{s}_{E}\left\{M_{1}^{\theta}\left(\Theta^{02} \cos \phi-\Theta^{01} \sin \phi\right)\right\}\right],
$$

\section{For Pair Production:}

Finally the differential cross section for incoming $\gamma$ beams in $(\mathrm{L}, \mathrm{R})$ state is given by

$$
\begin{gathered}
\left(\frac{d \sigma}{d \Omega}\right)_{\vec{\Theta}_{E}}=\frac{2 \alpha^{2}}{s}\left[\left(1+\cos ^{2} \theta\right) \csc ^{2} \theta-\bar{s}_{E}\left\{N_{1}^{\theta}\left(\Theta^{02} \cos \phi-\Theta^{01} \sin \phi\right)\right\}\right], \\
\bar{s}_{E}=\frac{s}{\Lambda_{E}^{2}} \quad M_{1}^{\theta}=\cot \theta \quad N_{1}^{\theta}=\frac{\csc \theta}{2}\left(1+\cos ^{2} \theta\right)
\end{gathered}
$$

The pure QED results can be recovered from the above expressions in the $\Lambda_{E}, \Lambda_{B} \rightarrow \infty$ limit. Since it is difficult to get time dependent data, we average over full day to be compared with the experiment. We will use following cross section observables to examine the effects of non commutativity

$$
\left\langle\frac{d \sigma}{d \cos \theta d \phi}\right\rangle_{T} \equiv \frac{1}{T_{d a y}} \int_{0}^{T_{d a y}} \frac{d \sigma}{d \cos \theta d \phi} d t
$$




$$
\begin{aligned}
\left\langle\frac{d \sigma}{d \cos \theta}\right\rangle_{T} & \equiv \frac{1}{T_{d a y}} \int_{0}^{T_{d a y}} \frac{d \sigma}{d \cos \theta} d t \\
\left\langle\frac{d \sigma}{d \phi}\right\rangle_{T} & \equiv \frac{1}{T_{d a y}} \int_{0}^{T_{d a y}} \frac{d \sigma}{d \phi} d t \\
\langle\sigma\rangle_{T} & \equiv \frac{1}{T_{d a y}} \int_{0}^{T_{d a y}} \sigma d t
\end{aligned}
$$

where

$$
\begin{aligned}
\frac{d \sigma}{d \cos \theta} & \equiv \int_{0}^{2 \pi} d \phi \frac{d \sigma}{d \cos \theta d \phi} \\
\frac{d \sigma}{d \phi} & \equiv \int_{-1}^{1} d(\cos \theta) \frac{d \sigma}{d \cos \theta d \phi} \\
\sigma & \equiv \int_{-1}^{1} d(\cos \theta) \int_{0}^{2 \pi} d \phi \frac{d \sigma}{d \cos \theta d \phi}
\end{aligned}
$$

However in the case of Pair annihilation, final state photons are identical, so one counts all the possible final states by integrating only over $0<\theta \leq \pi / 2$.

The initial phase dependence(i.e. $\xi$ ) disappears in time averaged observables and thus one can easily deduce $\{\Theta, \eta\}$ from them.

\section{NUMERICAL RESULTS}

In this section we will provide the numerical results of our investigation. In order to determine $\left\{\vec{\Theta}_{E}, \vec{\Theta}_{B}\right\}$ in laboratory system we studied variation of time averaged and time dependent cross section observables on $\{\xi, \eta, \Lambda\}$. We fixed the initial beam energy at $\sqrt{s}\left(=E_{\text {com }}\right)=800 \mathrm{GeV}$. The position of Lab system is fixed by taking $\delta=\pi / 4$ and $a=\pi / 4$.

The NC corrections in Pair annihilation and Pair creation, the $\phi$ dependence only appears in the form of $\cos \phi$ and $\sin \phi$. So non commutative effects can be obtained only in azimuthal angle distribution cross section $(d \sigma / d \phi)$ since they disappear in other observables once we integrate over the full azimuthal angle $(0-2 \pi)$. Hence for studying other cross section observables of these two processes we applied a cut of $(0-\pi)$ on azimuthal angle $\phi$.

Figs. 5-8 represents the variation of different cross section observables for Compton, Figs. 9-12 for Pair annihilation and Figs. 13-16 for Pair production case. 


\section{A. Time Averaged Angular distributions}

Non commutativity of space time defined by Eq,1 breaks Lorentz invariance including rotational invariance around the beam axis. This will lead to dependence of cross section on azimuthal angle which is absent in Standard Model. Thus non commutativity of space time can provide clear and distinct signature in azimuthal angular variation of cross sections.

In this section we will discuss the time averaged azimuthal $\left(\langle d \sigma / d \phi\rangle_{T}\right)$ and total cross section $\left(\langle\sigma\rangle_{T}\right)$ for different values of $\{\Lambda, \eta\}$. Our results are useful for case $s / \Lambda^{2}<1$ since in this domain one can safely ignore higher order corrections to cross section.

The angle $\eta$ can be determined by fitting the shape of curve of $(d \sigma / d \phi)_{T}$ plotted for different values of $\eta$ for a fixed value of non commutative scale especially around $\phi=$ $2 \pi / 3,5 \pi / 3$ in Compton and $\phi=6 \pi / 5, \pi / 5$ in Pair annihilation and Pair production case where respectively there is maximum enhancement and deficit in cross section compared to pure QED case. Similarly magnitude of NC parameter can be determined from fitting the curve of $(d \sigma / d \phi)_{T}$ plotted for different values of $\Lambda$ for a fixed $\eta$. For a fixed center of mass $\operatorname{energy}(\sqrt{s})$ the deviations to QED cross section becomes larger and larger as one lowers the value of non commutative $\operatorname{scale}(\Lambda)$.

Since NC corrections in time averaged cross sections for all three cases are proportional to $\cos \eta$ so their effect becomes maximum at $\eta=0, \pi$. Also correction is equal and opposite in magnitude for $\pi-\eta$ case. Thus polarization of beams is more useful compared to unpolarized case [39] where there is a two fold ambiguity in determination of $\eta$ for Pair annihilation.

The time averaged total cross section is also sensitive to the $|\Theta|$ and $\eta$. Thus one can also infer the information about the magnitude of NC parameter by fitting the $\left(\langle\sigma\rangle_{T}\right)$ curve plotted vs $\eta$ for different values of NC scale especially around $\eta=0, \pi$ where respectively there is maximum enhancement and deficit for Compton while opposite for Pair annihilation and Pair production.

Hence one can determine $\left\{\eta_{B}, \Theta_{B}\right\}$ from figures pertaining to Compton while $\left\{\eta_{E}, \Theta_{E}\right\}$ can be obtained from the Pair annihilation and Pair production curves without any ambiguity. 


\section{B. Time Dependent total Cross section}

In order to obtain angle $\xi$, we have studied the time variation of total cross section vs $\omega t-\xi$ with different values of $\eta$.

Figs. 8,12,16 gives the variation of $\sigma$ vs $\omega t-\xi$ for $\Lambda=1 \mathrm{TeV}$ with different values of $\eta$. It is clear from them that the cases with different $\eta$ are clearly distinguishable from each other. If the time variation of total cross section is observed then we can determine the magnitude

and direction of $\{\vec{\Theta}\}$ from the curves in terms of three parameters $\{\Theta, \eta, \xi\}$. The $\{\Theta, \eta\}$ can be obtained by fitting the magnitude and shape of curves while $\xi$ can be determined from the phase of time evolution of $\sigma$.

Although $\xi$ can be determined from the time variation of differential cross sections instead of total cross section however one can imagine that to plan such type of experiment needs very large luminosity because we must divide not only the phase space but also the time distribution into many bins, in order to get such dependence.

This completes our discussion for the determination of direction and magnitude of electric and magnetic components $\left(\left\{\vec{\Theta}_{E}, \vec{\Theta}_{B}\right\}\right)$ of NC parameter $\Theta$.

\section{SUMMARY AND DISCUSSION}

The NCSM is one of the extension for Physics beyond SM with motivations from string theory and quantum gravity. Its phenomenological implications are quite interesting since scale of non commutativity could be as low as a few $\mathrm{TeV}$, which can be explored at present or future colliders.

In the present work we have investigated the $\mathrm{TeV}$ scale signatures of $\mathrm{NC}$ space-time in $e^{+} e^{-} \rightarrow \gamma \gamma, e^{-} \gamma \rightarrow e^{-} \gamma$ and $\gamma \gamma \rightarrow e^{+} e^{-}$processes. We have done our study with initial beam polarization effects which offers the unique opportunity of having deviations from the SM cross sections occur at $\mathrm{O}(\Theta)$. Previous studies are mainly done with unpolarized case where these effects appear at $\mathrm{O}\left(\Theta^{2}\right)$. In this analysis we have also taken into account the apparent time variation of non commutative parameter $\left(\Theta^{\mu \nu}\right)$ in Laboratory frame. The primary coordinate system is fixed to the celestial sphere.

The NC corrections to Compton are sensitive to the magnetic component $\left(\vec{\Theta}_{B}\right)$ while for Pair production and Pair annihilation can probe electric component $\left(\vec{\Theta}_{E}\right)$ of $\mathrm{NC}$ parameter 
$(\vec{\Theta})$. Since such theories breaks rotational invariance around the beam axis, it leads to dependence of cross section on azimuthal angle which is absent in Standard Model. Thus they can provide clear and completely distinguishable signatures in azimuthal angular variation of cross sections.

To determine $\vec{\Theta}$ we have studied variation of various cross sections observables. Magnitude $|\vec{\Theta}|$ and angle $\eta$ can be determined by fitting the shape of curves of $(d \sigma / d \phi)$ plotted for different values of $\eta$ and $\Lambda$ respectively. From time variation of total cross section one

can determine magnitude as well as direction of NC parameter i.e. $\xi, \eta$ and $|\vec{\Theta}|$. These implications of non commutative space time can be tested at proposed International Linear Collider(ILC).

In this study for illustration purposes Lab coordinates are taken to be $(\delta, a)=(\pi / 4, \pi / 4)$. However in experiments with several detector sites such as LEP, the direction of incoming beam in each site is set to be along the different direction. Then angular distributions as well as time variation of observables will behave differently at each point because of the difference in direction of $\vec{\Theta}$ at different interacting points. Therefore combined analysis of various results from several experiments at different locations can help in probing the non commutative nature of space time.

\section{Acknowledgments}

S.K.G is grateful to Dr. Rolf Mertig for useful correspondence and Prof. B. Ananthanarayan for discussions. The work of N.G.D is supported by the US DOE under Grant No. DE-FG02-96ER40969. The work of P.K.D is supported by the DST Fast Track project SR/FTP/PS-11/2006 and BITS Seed Grant project, 2011. S.K.G acknowleges partial support from DST Ramanujan Fellowship SR/S2/RJN-25/2008. T.Shreecharan thanks UGC India for financial support through their Dr. D. S. Kothari Post-Doctoral Fellowship Scheme.

\section{APPENDIX A: COMPTON SCATTERING}

In this appendix we will reveal some details of our calculation. The complete $O(\Theta)$ Feynman amplitude square for Compton scattering is given by the expression: 


$$
\begin{aligned}
\mathcal{M \mathcal { M } ^ { \dagger }} & =\left(\mathcal{M M}^{\dagger}\right)^{C M}+\left(\mathcal{M M}^{\dagger}\right)_{C M}^{N C} \\
& =e^{4}\left\{T_{11} T_{11}^{\dagger}+T_{21} T_{21}^{\dagger}+T_{21} T_{11}^{\dagger}+T_{11} T_{21}^{\dagger}\right\} \epsilon_{r^{\prime}}^{*}\left(k^{\prime}\right) \epsilon_{r}(k) \epsilon_{r^{\prime}}\left(k^{\prime}\right) \epsilon_{r}^{*}(k) \\
& +\frac{i e^{4}}{2}\left\{T_{11}\left(T_{12}^{\Theta^{\dagger}}+T_{13}^{\Theta^{\dagger}}\right)+T_{21}\left(T_{22}^{\Theta^{\dagger}}+T_{23}^{\Theta^{\dagger}}\right)+T_{11}\left(T_{22}^{\Theta^{\dagger}}+T_{23}^{\Theta^{\dagger}}\right)+T_{21}\left(T_{12}^{\Theta^{\dagger}}+T_{11}^{\Theta^{\dagger}}\right)\right. \\
& +i T^{\Theta}\left(T_{11}^{\dagger}+T_{21}^{\dagger}\right)-\left(T_{12}^{\Theta}+T_{13}^{\Theta}\right) T_{11}^{\dagger}-\left(T_{22}^{\Theta}+T_{23}^{\Theta}\right) T_{21}^{\dagger}-\left(T_{22}^{\Theta}+T_{23}^{\Theta}\right) T_{11}^{\dagger} \\
& \left.-\left(T_{12}^{\Theta}+T_{13}^{\Theta}\right) T_{21}^{\dagger}+i\left(T_{11}+T_{21}\right) T^{\Theta \dagger}\right\} \epsilon_{r^{\prime}}^{*}\left(k^{\prime}\right) \epsilon_{r}(k) \epsilon_{r^{\prime}}\left(k^{\prime}\right) \epsilon_{r}^{*}(k) \\
& +O\left(\Theta^{2}\right) \text { terms }+\ldots \ldots \ldots .
\end{aligned}
$$

Here CM denotes the pure commutative part and $\mathrm{NC}-\mathrm{CM}$ is $O(\Theta)$ corrected terms arising due to interference between Commutative and Non commutative part. The various involved T terms are given by

$$
\begin{aligned}
\left(T_{11}\right)^{\mu \nu} & =\left[\bar{u}\left(p^{\prime}\right) H_{(L, R)} \gamma^{\mu} \frac{i}{\not p+\not k} \gamma^{\nu} u(p)\right] \\
\left(T_{12}^{\Theta}\right)^{\mu \nu} & =\left[\bar{u}\left(p^{\prime}\right) H_{(L, R)}\left(\Gamma_{12}^{\Theta}\right)^{\mu} \frac{i}{\not p+\not k} \gamma^{\nu} u(p)\right] \\
\left(T_{13}^{\Theta}\right)^{\mu \nu} & =\left[\bar{u}\left(p^{\prime}\right) H_{(L, R)} \gamma^{\mu} \frac{i}{\not p+\not k}\left(\Gamma_{13}^{\Theta}\right)^{\nu} u(p)\right] \\
\left(T_{21}\right)^{\mu \nu} & =\left[\bar{u}\left(p^{\prime}\right) H_{(L, R)} \gamma^{\mu} \frac{i}{\not p-\not k^{\prime}} \gamma^{\nu} u(p)\right] \\
\left(T_{22}^{\Theta}\right)^{\mu \nu} & =\left[\bar{u}\left(p^{\prime}\right) H_{(L, R)}\left(\Gamma_{22}^{\Theta}\right)^{\mu} \frac{i}{\not p-\not k^{\prime}} \gamma^{\nu} u(p)\right] \\
\left(T_{23}^{\Theta}\right)^{\mu \nu} & =\left[\bar{u}\left(p^{\prime}\right) H_{(L, R)} \gamma^{\mu} \frac{i}{\not p-\not k^{\prime}}\left(\Gamma_{23}^{\Theta}\right)^{\nu} u(p)\right] \\
\left(T^{\Theta}\right)^{\mu \nu} & =\left[\bar{u}\left(p^{\prime}\right) \Theta^{\mu \nu \rho}\left(k^{\prime}+k\right)_{\rho} H_{(L, R)} u(p)\right]
\end{aligned}
$$

where different $\Gamma^{\Theta}$ terms are given by 


$$
\begin{aligned}
& \left(\Gamma_{13}^{\Theta}\right)^{\mu}=((p+k) \Theta p) \gamma^{\mu}-((p+k) \Theta)^{\mu} \not p-(\not p+\not k)(\Theta p)^{\mu} \\
& \left(\Gamma_{12}^{\Theta}\right)^{\mu}=\left(p^{\prime} \Theta(p+k)\right) \gamma^{\mu}-\left(p^{\prime} \Theta\right)^{\mu}(\not p+\not k)-\not p^{\prime}(\Theta(p+k))^{\mu} \\
& \left(\Gamma_{23}^{\Theta}\right)^{\mu}=\left(\left(p-k^{\prime}\right) \Theta p\right) \gamma^{\mu}-\left(\left(p-k^{\prime}\right) \Theta\right)^{\mu} \not p-\left(\not p-\not k^{\prime}\right)(\Theta p)^{\mu} \\
& \left(\Gamma_{22}^{\Theta}\right)^{\mu}=\left(p^{\prime} \Theta\left(p-k^{\prime}\right)\right) \gamma^{\mu}-\left(p^{\prime} \Theta\right)^{\mu}\left(\not p-\not k^{\prime}\right)-\not p^{\prime}\left(\Theta\left(p-k^{\prime}\right)\right)^{\mu}
\end{aligned}
$$

Using these expressions one can put the different terms of $\mathcal{M M}^{\dagger}$ in form of trace expressions. e.g.

$$
\begin{aligned}
T_{11} T_{11}^{\dagger} & =\frac{1}{(p+k)^{4}} \operatorname{Tr}\left\{\not p^{\prime} H_{(L, R)} \gamma^{\mu}(\not p+\not k) \gamma^{\nu} \not p \gamma^{b}(\not p+\not k) \gamma^{a}\right\} \\
T_{21} T_{21}^{\dagger} & =\frac{1}{\left(p-k^{\prime}\right)^{4}} \operatorname{Tr}\left\{\not p^{\prime} H_{(L, R)} \gamma^{\nu}\left(\not p-\not k^{\prime}\right) \gamma^{\mu} \not p \gamma^{a}\left(\not p-\not k^{\prime}\right) \gamma^{b}\right\} \\
T_{12}^{\Theta} T_{11}^{\dagger} & =\frac{1}{(p+k)^{4}} \operatorname{Tr}\left\{\not p H_{(L, R)}\left(X_{12}^{\Theta}\right)^{\mu}(\not p+\not k) \gamma^{\nu} \not p \gamma^{b}(\not p+\not k) \gamma^{a}\right\} \\
T_{13}^{\Theta} T_{21}^{\dagger} & =\frac{1}{(p+k)^{2}\left(p-k^{\prime}\right)^{2}} \operatorname{Tr}\left\{\not \phi^{\prime} H_{(L, R)} \gamma^{\mu}(\not p+\not k)\left(X_{13}^{\Theta}\right)^{\nu} \not p \gamma^{a}\left(\not p-\not k^{\prime}\right) \gamma^{b}\right\}
\end{aligned}
$$

with

$$
\begin{aligned}
& \left(X_{12}^{\Theta}\right)^{\mu}=C_{2} \gamma^{\mu}-\left(V_{3}\right)^{\mu}(\not p+\not k)+\not p^{\prime}\left(V_{1}\right)^{\mu} \\
& \left(X_{13}^{\Theta}\right)^{\mu}=C_{1} \gamma^{\mu}-\left(V_{1}\right)^{\mu} \not p-(\not p+\not k)\left(V_{2}\right)^{\mu}
\end{aligned}
$$

Here

$$
\begin{aligned}
& C_{1}=(p+k) \Theta p ; \quad C_{2}=p^{\prime} \Theta(p+k) \\
& V_{1}=(p+k) \Theta ; \quad V_{2}=\Theta p ; \quad V_{3}=p^{\prime} \Theta
\end{aligned}
$$

Then these trace expressions can be evaluated using the Mathematica packages like FeynCalc[54] or Symbolic Manipulation programme FORM[55]. In this way one can obtain various cross section observables.

[1] N. Seiberg and E. Witten, JHEP 9909, 032 (1999) arXiv:hep-th/9908142. 
[2] H. S. Snyder, Phys. Rev. 71, 38 (1947).

[3] S. Minwalla, M. Van Raamsdonk and N. Seiberg, JHEP 0002, 020 (2000) arXiv:hep-th/9912072.

[4] A. Matusis, L. Susskind and N. Toumbas, JHEP 0012, 002 (2000) arXiv:hep-th/0002075.

[5] L. Alvarez-Gaume and M. A. Vazquez-Mozo, Nucl. Phys. B 668, 293 (2003) arXiv:hep-th/0305093.

[6] J. Jaeckel, V. V. Khoze and A. Ringwald, JHEP 0602, 028 (2006) arXiv:hep-ph/0508075.

[7] S. A. Abel, J. Jaeckel, V. V. Khoze and A. Ringwald, JHEP 0609, 074 (2006) arXiv:hep-ph/0607188.

[8] R. Horvat and J. Trampetic, JHEP 1101, 112 (2011) arXiv:1009.2933 [hep-ph]].

[9] B. Jurco, S. Schraml, P. Schupp and J. Wess, Eur. Phys. J. C 17, 521 (2000) arXiv:hep-th/0006246].

[10] B. Jurco, L. Moller, S. Schraml, P. Schupp and J. Wess, Eur. Phys. J. C 21, 383 (2001) arXiv:hep-th/0104153.

[11] X. Calmet, B. Jurco, P. Schupp, J. Wess and M. Wohlgenannt, Eur. Phys. J. C 23, 363 (2002) arXiv:hep-ph/0111115.

[12] W. Behr, N. G. Deshpande, G. Duplancic, P. Schupp, J. Trampetic and J. Wess, Eur. Phys. J. C 29, 441 (2003) arXiv:hep-ph/0202121.

[13] G. Duplancic, P. Schupp and J. Trampetic, Eur. Phys. J. C 32, 141 (2003) arXiv:hep-ph/0309138.

[14] T. Ohl and J. Reuter, Phys. Rev. D 70, 076007 (2004) arXiv:hep-ph/0406098.

[15] B. Melic, K. Passek-Kumericki, J. Trampetic, P. Schupp and M. Wohlgenannt, Eur. Phys. J. C 42, 483 (2005) arXiv:hep-ph/0502249.

[16] B. Melic, K. Passek-Kumericki, J. Trampetic, P. Schupp and M. Wohlgenannt, Eur. Phys. J. C 42, 499 (2005) arXiv:hep-ph/0503064.

[17] P. K. Das, N. G. Deshpande and G. Rajasekaran, Phys. Rev. D 77, 035010 (2008) arXiv:0710.4608 [hep-ph]].

[18] J.Hewett, F.J.Petriello and T.G.Rizzo, hep-ph/0201275 J.Hewett et al., Phys. Rev. D 64, 075012 (2001), Phys. Rev. D 66, 036001 (2002).

[19] P. Schupp, J. Trampetic, J. Wess and G. Raffelt, "The photon neutrino interaction in noncommutative gauge field theory and Eur. Phys. J. C 36, 405 (2004) arXiv:hep-ph/0212292. 
[20] M. Haghighat, M. M. Ettefaghi and M. Zeinali, Phys. Rev. D 73, 013007 (2006) arXiv:hep-ph/0511042.

[21] M. Mohammadi Najafabadi, "Semi-Leptonic Decay of a Polarized Top Quark in the Noncommutative Standard Phys. Rev. D 74, 025021 (2006) arXiv:hep-ph/0606017].

[22] N. Mahajan, Phys. Rev. D 68, 095001 (2003) arXiv:hep-ph/0304235.

[23] E. O. Iltan, "The $Z^{-} \rightarrow \ell^{+} \ell^{-}$and $W \rightarrow \nu_{\ell} \ell^{+}$decays in Phys. Rev. D 66, 034011 (2002) arXiv:hep-ph/0204332.

[24] N. G. Deshpande and X. G. He, Phys. Lett. B 533, 116 (2002) arXiv:hep-ph/0112320.

[25] M. M. Najafabadi, Phys. Rev. D 77, 116011 (2008) arXiv:0803.2340 [hep-ph]].

[26] J. Trampetic, "Renormalizability and Phenomenology of theta-expanded Noncommutative Gauge Fortsch. Phys. 56, 521 (2008) [arXiv:0802.2030 [hep-ph]].

[27] M. Buric, D. Latas, V. Radovanovic and J. Trampetic, Phys. Rev. D 75, 097701 (2007).

[28] G. Abbiendi et al. [OPAL Collaboration], Phys. Lett. B 568, 181 (2003) arXiv:hep-ex/0303035.

[29] B. Melic, K. Passek-Kumericki and J. Trampetic, "Quarkonia decays into two photons induced by the space-time Phys. Rev. D 72, 054004 (2005) arXiv:hep-ph/0503133].

[30] B. Melic, K. Passek-Kumericki and J. Trampetic, Phys. Rev. D 72, 057502 (2005) arXiv:hep-ph/0507231.

[31] A. Alboteanu, T. Ohl and R. Ruckl, Phys. Rev. D 76, 105018 (2007) arXiv:0707.3595 [hep$\mathrm{ph}]$.

[32] A. Prakash, A. Mitra and P. K. Das, Phys. Rev. D 82, 055020 (2010) arXiv:1009.3554 [hep$\mathrm{ph}]$.

[33] P. K. Das, A. Prakash and A. Mitra, Phys. Rev. D 83, 056002 (2011) arXiv:1009.3571 [hep$\mathrm{ph}]$.

[34] W. Wang, F. Tian and Z. M. Sheng, arXiv:1105.0252 [hep-ph].

[35] A. Alboteanu, T. Ohl and R. Ruckl, Phys. Rev. D 74, 096004 (2006) arXiv:hep-ph/0608155.

[36] A. Alboteanu, T. Ohl and R. Ruckl, eConf C0705302, TEV05 (2007) [Acta Phys. Polon. B 38, 3647 (2007)] [arXiv:0709.2359 [hep-ph]].

[37] H. Grosse and Y. Liao, Phys. Rev. D 64, 115007 (2001) arXiv:hep-ph/0105090.

[38] Y. Liao and C. Dehne, Eur. Phys. J. C 29, 125 (2003) arXiv:hep-ph/0211425].

[39] J. i. Kamoshita, Eur. Phys. J. C 52, 451 (2007) arXiv:hep-ph/0206223. 
[40] M. Haghighat, N. Okada and A. Stern, Phys. Rev. D 82, 016007 (2010) arXiv:1006.1009 [hep-ph]].

[41] J. (. ). Brau et al. [ILC Collaboration], arXiv:0712.1950 [physics.acc-ph].

[42] G. Aarons et al. [ILC Collaboration], arXiv:0709.1893 [hep-ph].

[43] A. Bichl, J. Grimstrup, H. Grosse, L. Popp, M. Schweda and R. Wulkenhaar, JHEP 0106, 013 (2001) arXiv:hep-th/0104097.

[44] C. P. Martin, Nucl. Phys. B 652, 72 (2003) arXiv:hep-th/0211164.

[45] C. P. Martin and C. Tamarit, Phys. Rev. D 72, 085008 (2005) arXiv:hep-th/0503139.

[46] M. Buric, V. Radovanovic and J. Trampetic, JHEP 0703, 030 (2007) arXiv:hep-th/0609073].

[47] D. Latas, V. Radovanovic and J. Trampetic, Phys. Rev. D 76, 085006 (2007) [arXiv:hep-th/0703018].

[48] M. Buric, D. Latas, V. Radovanovic and J. Trampetic, Phys. Rev. D 77, 045031 (2008) arXiv:0711.0887 [hep-th]].

[49] C. P. Martin and C. Tamarit, Phys. Lett. B 658, 170 (2008) [arXiv:0706.4052 [hep-th]].

[50] M. M. Ettefaghi, M. Haghighat and R. Mohammadi, Phys.Rev. D 82, 105017 (2010).

[51] M. Buric, D. Latas, V. Radovanovic and J. Trampetic, Phys. Rev. D 83, 045023 (2011) arXiv:1009.4603 [hep-th]].

[52] R. Horvat, D. Kekez and J. Trampetic, Phys. Rev. D 83, 065013 (2011) arXiv:1005.3209 [hep-ph]].

[53] R. Horvat, D. Kekez, P. Schupp, J. Trampetic and J. You, arXiv:1103.3383 [hep-ph].

[54] R. Mertig, M. Bohm and A. Denner, Comput. Phys. Commun. 64, 345 (1991).

[55] J. A. M. Vermaseren, arXiv:math-ph/0010025. 

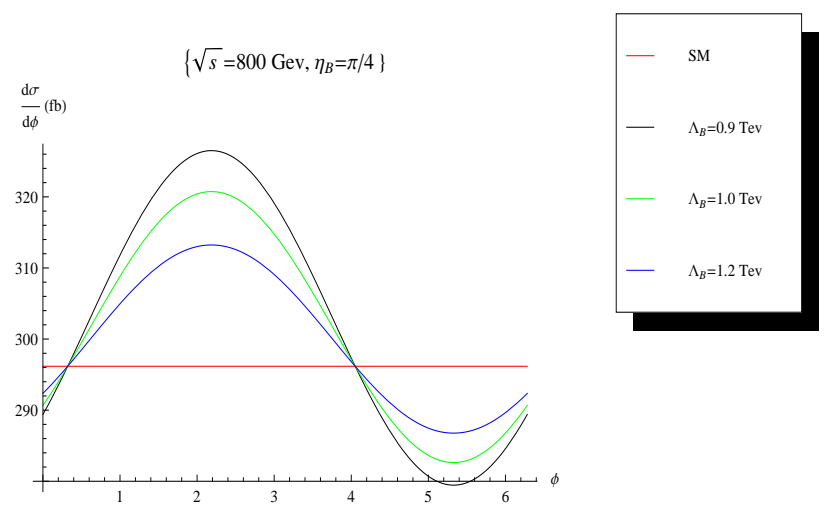

FIG. 5: Compton Scattering: Time averaged polar angle distribution vs $\phi$ for different values of $\mathrm{NC}$ scale $\left(\Lambda_{B}\right)$.
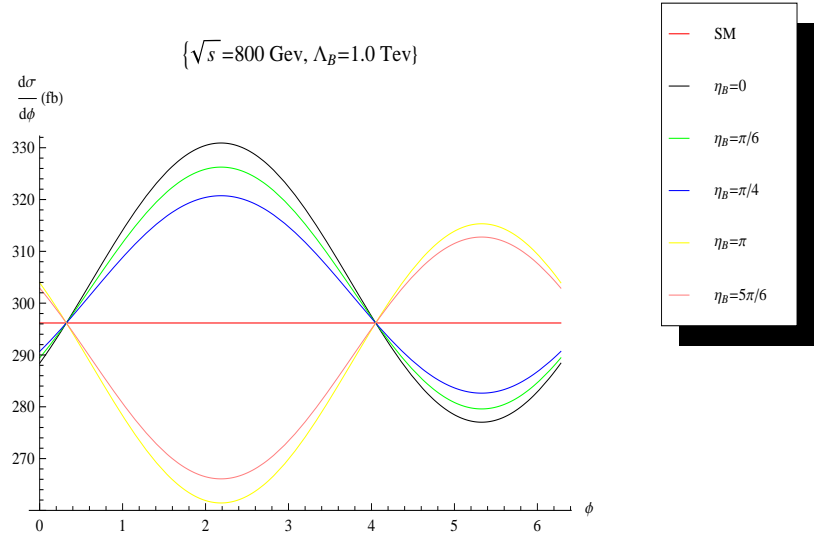

FIG. 6: Compton Scattering: Time averaged azimuthal angle distribution vs $\phi$ for different values of angle $\eta_{B}$.
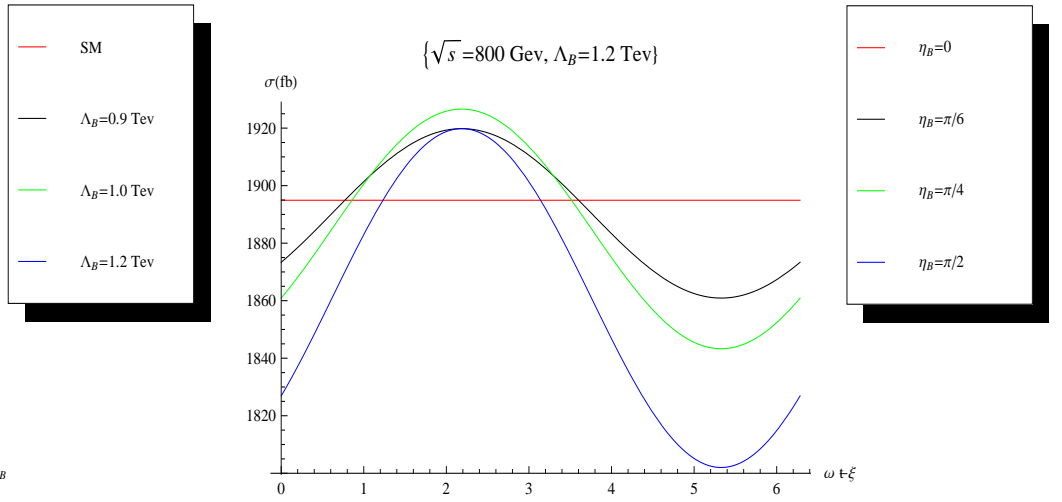

FIG. 7: Compton Scattering: Time average of FIG. 8: Compton Scattering: Time dependent total cross section $\left(\sigma_{T}\right)$ for different values of $\mathrm{NC}$ $\operatorname{scale}\left(\Lambda_{B}\right)$. total cross section $(\sigma)$ vs phase $(\omega t-\xi)$ for different values of angle $\eta_{B}$. 


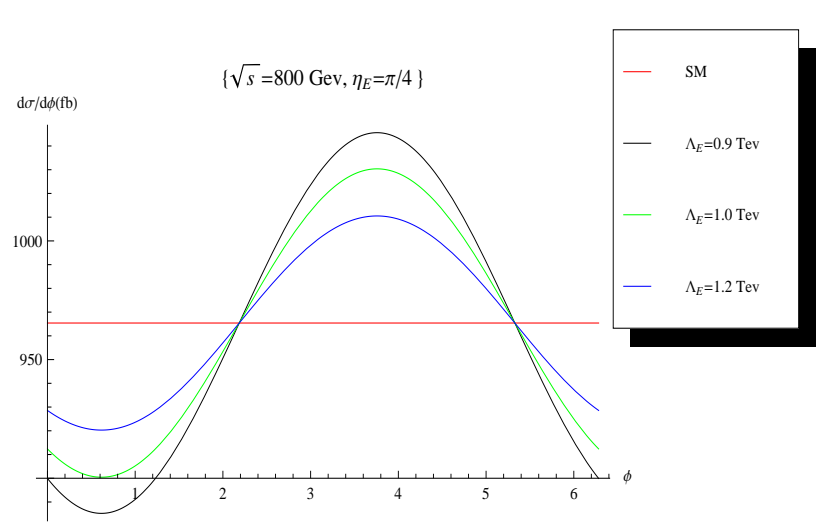

FIG. 9: Pair Production: Time averaged polar angle distribution vs $\phi$ for different values of $\mathrm{NC}$ $\operatorname{scale}\left(\Lambda_{E}\right)$.

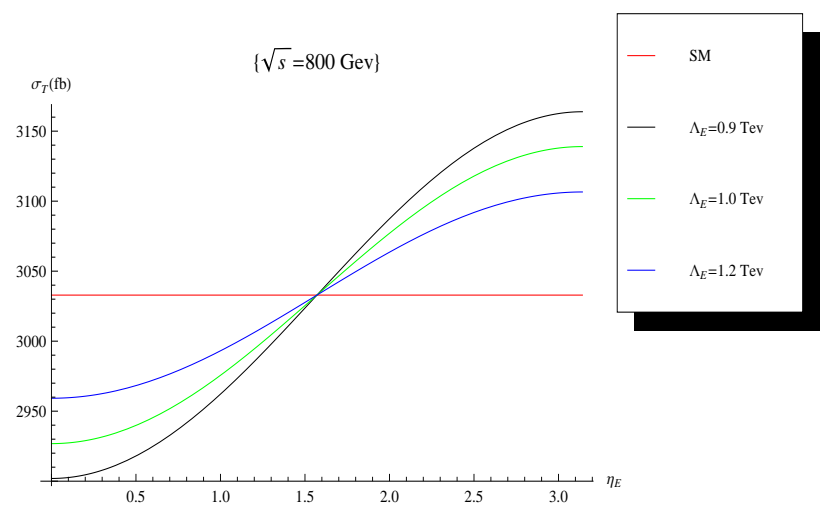

FIG. 11: Pair Production: Time average of total cross section $\left(\sigma_{T}\right)$ for different values of $\mathrm{NC}$ $\operatorname{scale}\left(\Lambda_{E}\right)$.

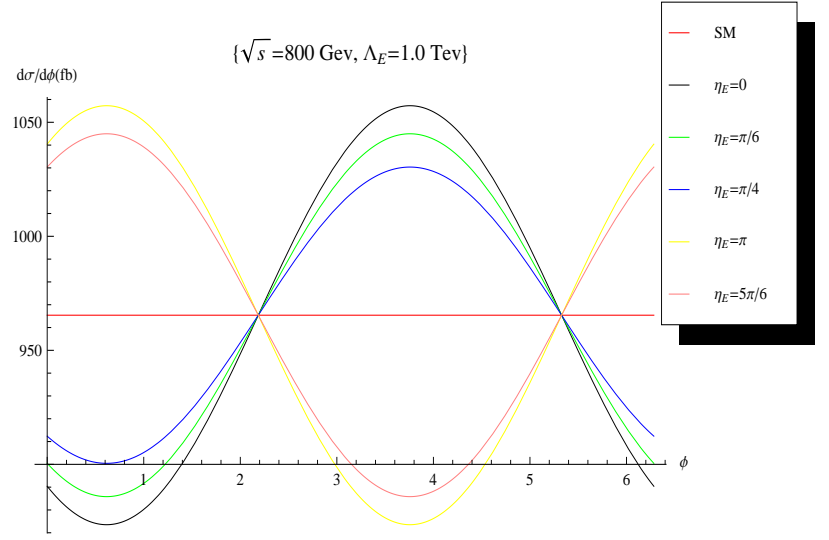

FIG. 10: Pair Production: Time averaged azimuthal angle distribution vs $\phi$ for different values of angle $\eta_{E}$.

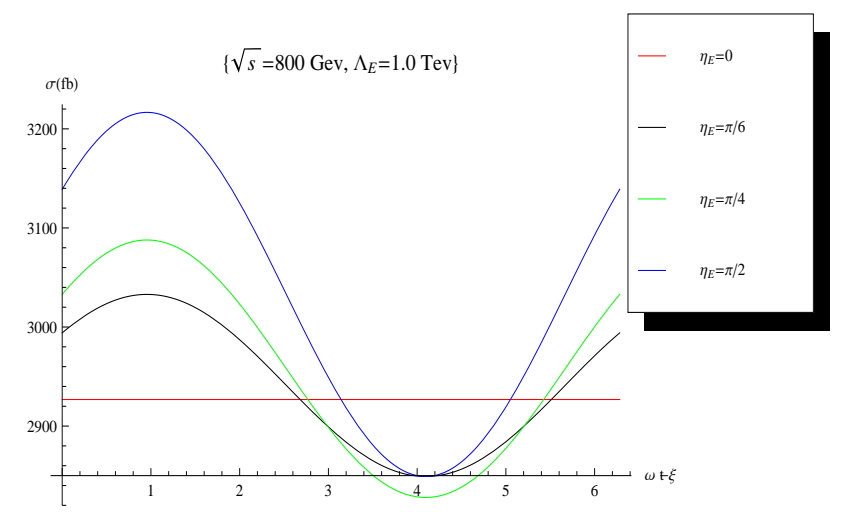

FIG. 12: Pair Production: Time dependent total cross section $(\sigma)$ vs phase $(\omega t-\xi)$ for different values of angle $\eta_{E}$. 

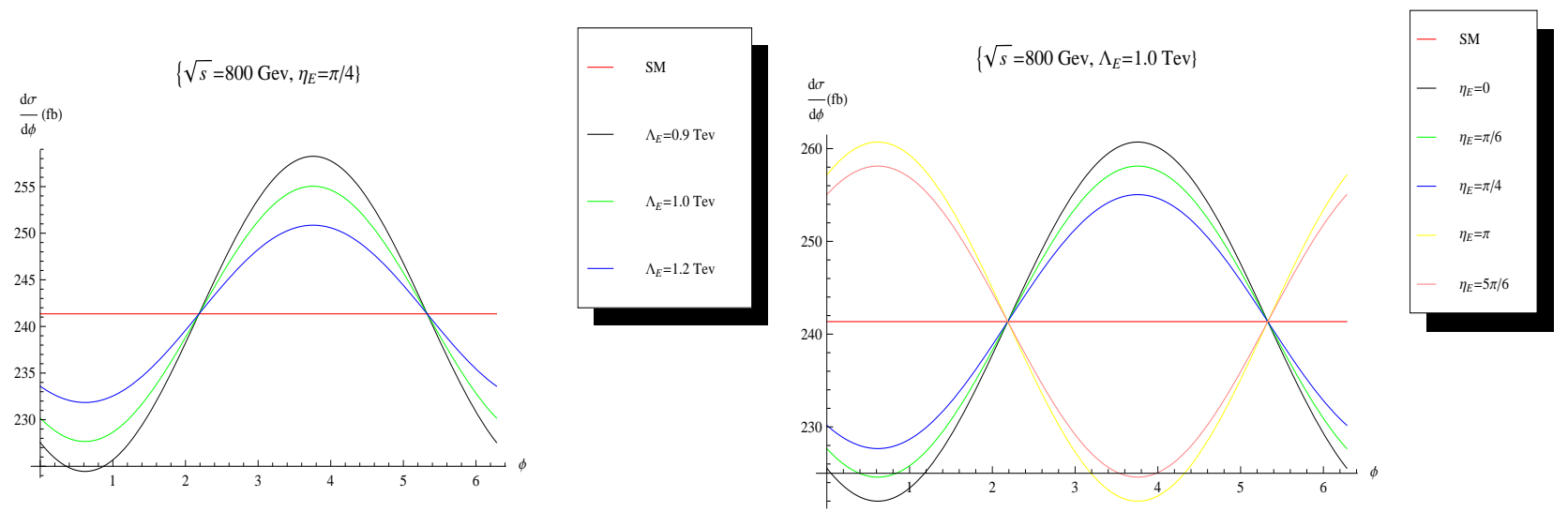

FIG. 13: Pair Annihilation: Time averaged poFIG. 14: Pair Annihilation: Time averaged azlar angle distribution vs $\phi$ for different values of $\mathrm{NC} \operatorname{scale}\left(\Lambda_{E}\right)$. imuthal angle distribution vs $\phi$ for different values of angle $\eta_{E}$.
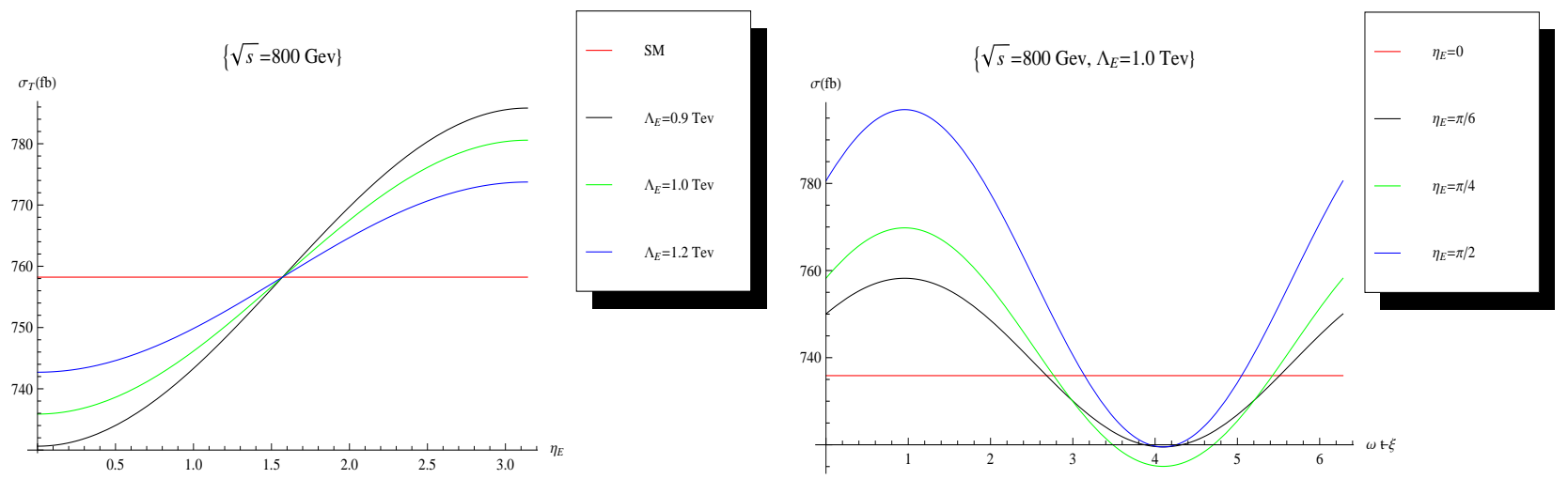

FIG. 15: Pair Annihilation: Time average of FIG. 16: Pair Annihilation: Time dependent tototal cross section $\left(\sigma_{T}\right)$ for different values of tal cross section $(\sigma)$ vs phase $(\omega t-\xi)$ for different $\mathrm{NC} \operatorname{scale}\left(\Lambda_{E}\right)$. values of angle $\eta_{E}$. 